\title{
On the origin of negative target currents during laser ablation of polyethylene
}

\author{
Domenico Delle Side ${ }^{1,2, \star}$, Anna Paola Caricato ${ }^{1,2}$, Josef Krása ${ }^{3}$, and Vincenzo Nassisi ${ }^{1,2}$ \\ ${ }^{1}$ Dipartimento di Matematica e Fisica “E. De Giorgi”, Universitá del Salento - Lecce, Italy \\ ${ }^{2}$ Istituto Nazionale di Fisica Nucleare, Sezione di Lecce - Lecce, Italy \\ ${ }^{3}$ Institute of Physics, ASCR - Praha 8, Czech Republic
}

\begin{abstract}
The exposure of a target to a focused laser beam results in the occurrence of a time-varying current between the target itself and the grounded vacuum chamber. This current is composed by three distinct phases, namely the ignition phase, in which the laser pulse drives the electron emission, while electrons coming from the ground through the target holder balance the positive charge generated on the target. The active phase appears at post-pulse times and it is characterized by the presence of peaked structures in the time-resolved current, representing characteristics of the target composition. Lastly, the afterglow phase is determined by a current of electrons flowing from the target to the ground. During the active phase of the target current resulting from polymers ablation with an $\mathrm{UV} \mathrm{KrF}$ laser, negative target current peaks are observed, whose origin is still unknown. We investigate the dependence of these current structures on the dimensions of the target, using ultra-high molecular weight polyethylene disks of different thickness.
\end{abstract}

\section{Introduction}

The interaction of an intense laser pulse with a solid target give rise to a series of complex phenomena that eventually ends with ejection of vaporized and ionized material from the target surface. Obviously, depending on the the laser pulse length, the physical processes underlying the ejection could change [1]. Neverthless, ejecta are readily created after the first moments of the laser pulse, both as neutral particles and ions, electrons being the first. Unless electrically insulated, the escape of charged particles from the target generates a return current from ground that balances such process. Target current (TC) is a relatively unexplored phenomenum in the field of laser-matter interaction. The initial studies started in the late seventies $[2,3]$ and continued in a patchy fashion across the following decades $[4,5]$ until recently.

In the last years, ultra-intense laser systems (from TW to PW powers) become available, leading to the discovery of the emission of hot electrons and energetic ions from the interaction of such laser with thin (from 1 to $10 \mu \mathrm{m}$ ) foils [6]. This renewed the interest in TC as tool to obtain insights both for the ion emission and the generated electromagnetic pulses [7-12].

Despite of the intriguing possibilities it offers, e.g. experimental study of electrons overcoming the plasma potential barrier and escaping the plasma, the physical description of TC in the context of such experiments is difficult, since currents reach values in the kA range, leading often to extremely complicated experimental setups. For such reasons, in 2014 we started an experimental cam-

\footnotetext{
^e-mail: domenico.delleside@le.infn.it
}

paign to analyze TC in the simpler framework of nanosecond laser ablation, in order to gain insight on the underlying process in a "relaxed" physical picture. We showed, in particular, that TC is composed by three distinct phases [13], namely the ignition phase, in which the laser pulse drives the electron emission, while electrons coming from the ground through the target holder balance the positive charge generated on the target. The active phase appears at post-pulse times and it is characterized by the presence of peaked structures in the time-resolved current, representing characteristics of the target composition. Lastly, the afterglow phase is determined by a current of electrons flowing from the target to the ground. Furthermore, changing the target material, we showed that the occurrence in time of current peak in the active phase is strongly related to the charge-to-mass ratio of the generated ion species [14], i.e.

$$
t_{P E A K} \propto\left(\frac{A}{Z}\right)^{1 / 2}
$$

where $Z$ represents charge state, while $A$ the mass number. The duration of target neutralization with ground electrons reflects the duration of electron escape from the expanding plasma [14]. The TC occurs when the laser pulse starts to produce the plasma [11, 13].

Target current registered during ablation of plastic targets revealed an unxpected and unexplained behavior. Indeed, during ablation of conductive targets, TC readily passes from zero to $\mathrm{mA}$ peak values during the ignition and active phases, switching to negative values during the afterglow phase. In plastic targets, instead, we found that $\mathrm{TC}$ assumes negative values during the ignition phase $[15,16]$. Furthermore, we showed that such negative 


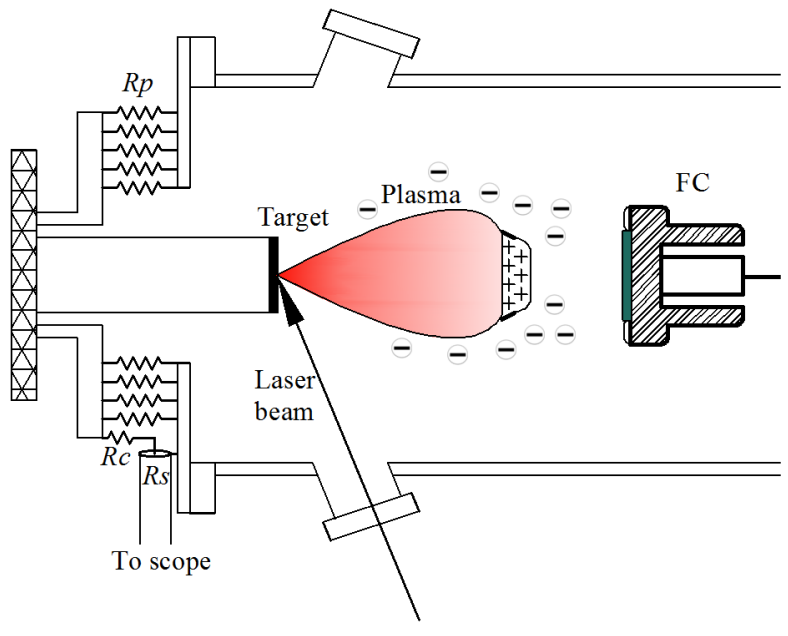

Figure 1. Sketch of the experimental setup: $R_{p}$ parallel resistors, $R_{c}$ single $60 \Omega$ resistor, $R_{s}$ scope impedance, FC is a Faraday cup.

current strongly depends on the target-holder-to-samplesurface ratio. This article is devoted to a deeper investigation of this obscure phenomenon.

\section{Materials and methods}

The experiments described here took place at LEAS Laboratory of Dipartimento di Matematica e Fisica "E. De Giorgi", Universitá del Salento, in Lecce (Italy). We used the PLATONE setup [17-19], a nanosecond Laser Ion Source operating in the UV range. Indeed, it uses a $\mathrm{KrF}$ excimer laser with pulse length of $23-25 \mathrm{~ns}$ and an intensity ranging from $10^{8}$ to $10^{10} \mathrm{~W} / \mathrm{cm}^{2}$ (laser spot diameter $\approx 180 \mu \mathrm{m}$ ), operated in single shot mode. During the experiments, we used a fluence of $40 \mathrm{~J} / \mathrm{cm}^{2}$, corresponding to an intensity of $\sim 1.7 \times 10^{9} \mathrm{~W} / \mathrm{cm}^{2}$. The target holder, initially insulated, was connected to ground through an array $R_{p}$ of 10 resistors $(110 \Omega)$. One of such resistors was the series between a further $60 \Omega$ resistor $\left(R_{c}\right)$ and a digital oscilloscope (see Figure 1). Consequently, the voltage $V_{S}$ registered on the scope is related to the potential jump on the laser target $\left(V_{T}\right)$ through the formula

$$
V_{T}(t)=\frac{R_{c}+R_{s}}{R_{S}} V_{S}(t)
$$

with $R_{S}$ being the scope internal impedance (i.e. $50 \Omega$ ). To diagnose the ion beams resulting from laser-matter interaction, we used a negatively biased Faraday cup (FC) with a grounded input mesh. FC was placed in front of the target $(\mathrm{T})$ at a distance of $285 \mathrm{~mm}$. Through a dedicated filter circuit, FC was connected to the same oscilloscope used to record the TC.

In order to gain insight on the origin of the negative peak observed in plastic materials, we used as targets commercial Ultra High Molecular Weight Polyethylene (UHMWPE) disks ( $20 \mathrm{~mm}$ diameter) of 4 different thickness: $1.2,0.5,0.2$ and $0.075 \mathrm{~mm}$.

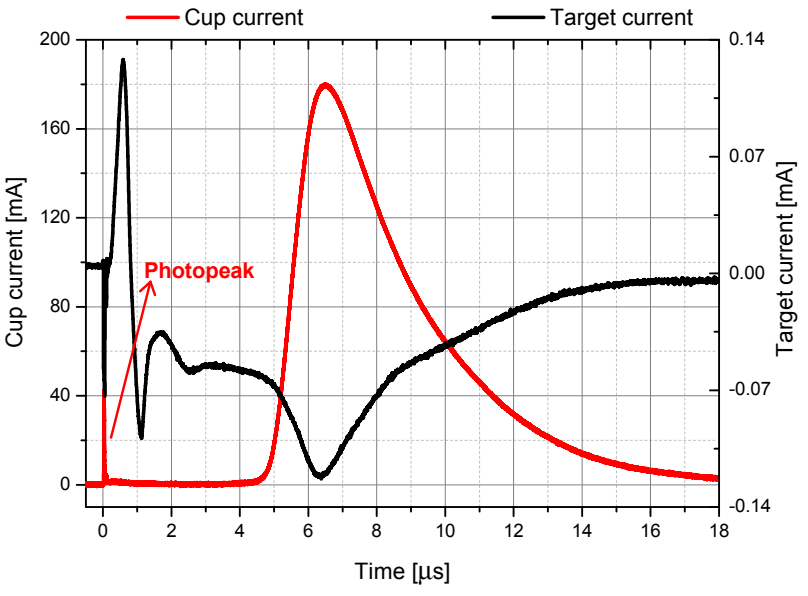

Figure 2. Representative results of both target current and the corresponding cup current measured on FC (target thickness is $0.2 \mathrm{~mm})$

\section{Results and Discussion}

The laser-matter interaction with the targets resulted in both TC and FC spectra, for all targets, that are consistent with the observation performed in the past $[15,16]$ (see Figure 2). As expected, the TC presented negative peaks for all the target thickness used. High resolution spectra of TC showed that in all samples, the negative part is composed of two distinct peaks (P1 occurring near $20 \mathrm{~ns}$ and P2 after 55 ns, see Figure 3). Despite of this, the temporal structure and the amplitude of the signals changed significantly from sample to sample. First of all, considering only signal amplitude, it appears that it exists an optimal thickness able to increase the height of the resulting TC peaks, as shown in Figure 3. Indeed, the maximum amplitude happens for a thickness of $0.5 \mathrm{~mm}$, decreasing moving both to the left and right of this value. We'll explain such behavior below.

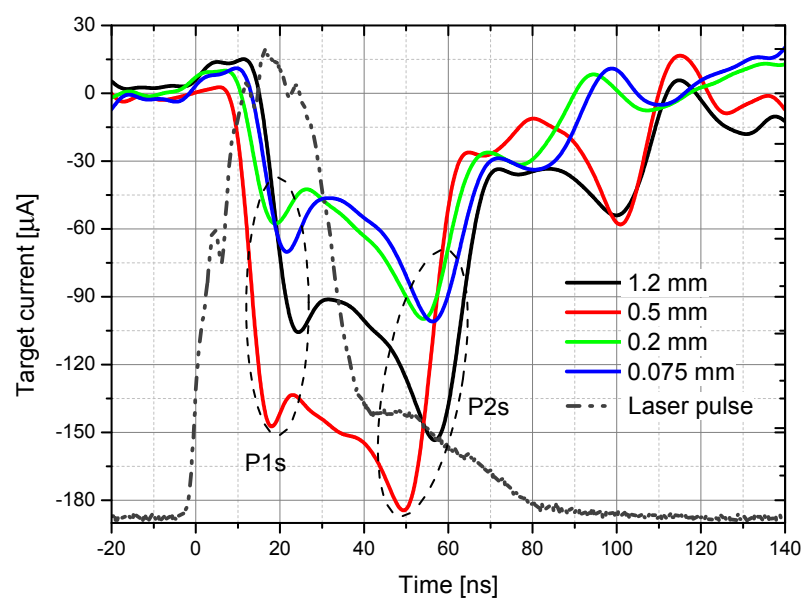

Figure 3. Details of the negative current peaks for all the targets thickness used, together with a waveform representing the laser pulse. The two ellipses identify P1 and P2 for each curve. 

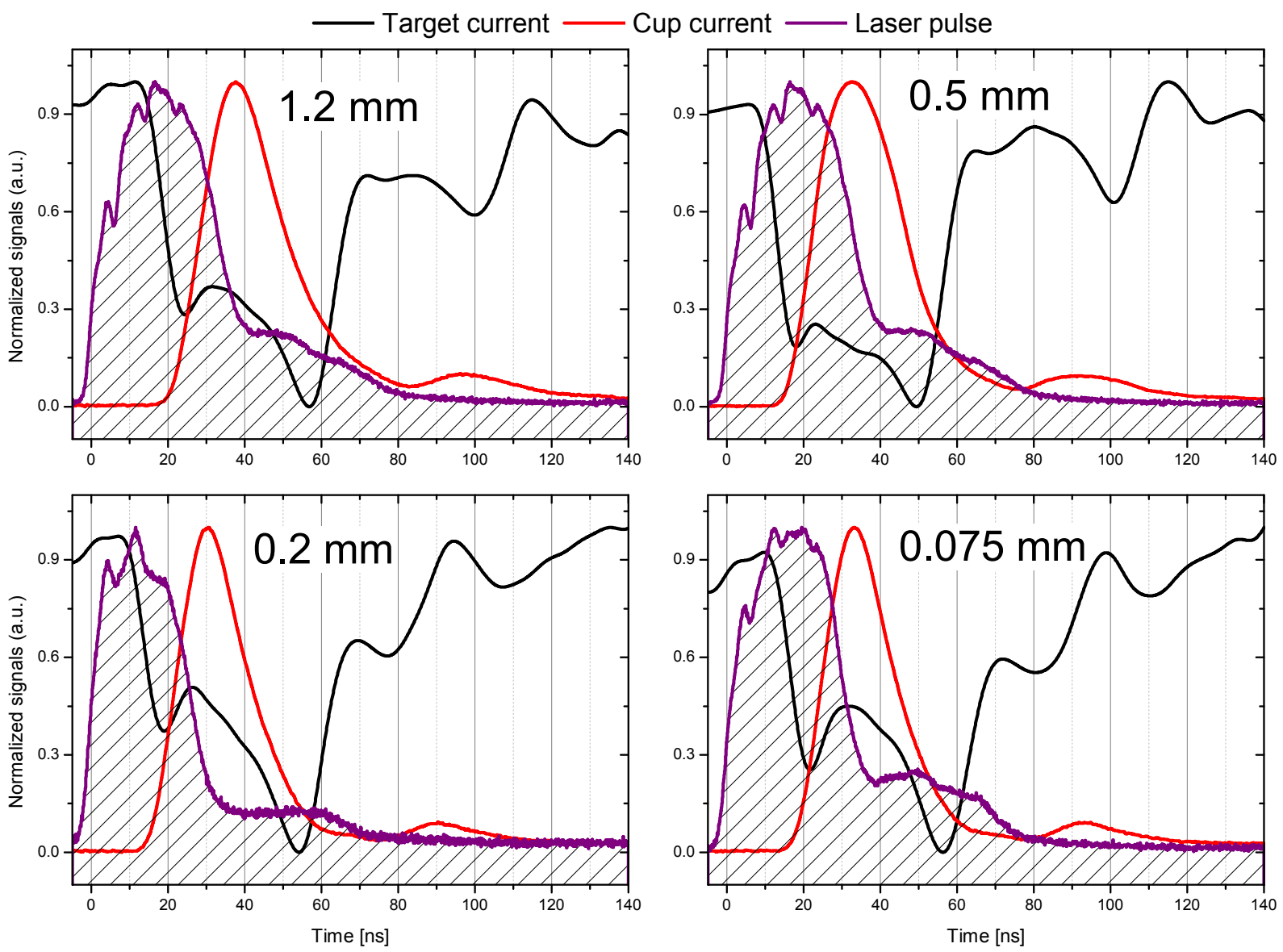

Figure 4. Normalized signals of TC, FC and laser pulse for the 4 targets under exam. It is worth noticing that for practical reasons, TC refers to the left scale, while FC current and the laser pulse to the right. Both scales run from 0 to 1 , but the left, to maximize the TC peaks, stops slightly before 1 .

The temporal occurrence of the negative peaks suggests a possible involvement of the laser-plasma XUV and soft X-rays that give rise to the photopeak signal on FC. For this reason, we plot on the same figure the results obtained for the 4 targets, normalizing TC, the photopeak and the laser pulse to the same $[0,1]$ scale (Figure 4).

Such comparison shows that the first peak occurs within the end of the laser pulse and the onset of the photopeak. This suggests that P1 is intrinsically related with the lasermatter interaction process. Such behavior could be explained considering the role of target electrons heated during the action of the laser pulse. Indeed, electrons absorbing the $248 \mathrm{~nm}$ laser radiation which penetrates into the plastic foil and also plasma radiation are liberated in the target via internal photoemission. These electrons could then diffuse to the target holder, flowing to ground and giving rise to negative currents. This explanation is also at the origin of the "optimal thickness" effect observed on the top of this section. Such thickness resulted about $0.5 \mathrm{~mm}$ in our experiments. Due to the thermal nature of $\mathrm{P} 1$, when target is too thin, it cools too rapidly, leading to smaller current amplitudes. Conversely, when the thickness is too large, the electron stream reaching the target holder is reduced, since they encounter more recombination centers. Also in the latter case, we obtain smaller current ampli- tudes. The nature of $\mathrm{P} 2$, instead, remains still unclear from the comparison. In order to obtain a clearer picture of the situation, we performed measurements of TC changing the negative bias on FC, leaving the grounded input mesh in its position. The increase (in absolute value) of polarization induced a sensible increase on P2's amplitude and a shift of its position towards P1, as shown in Figure 5.

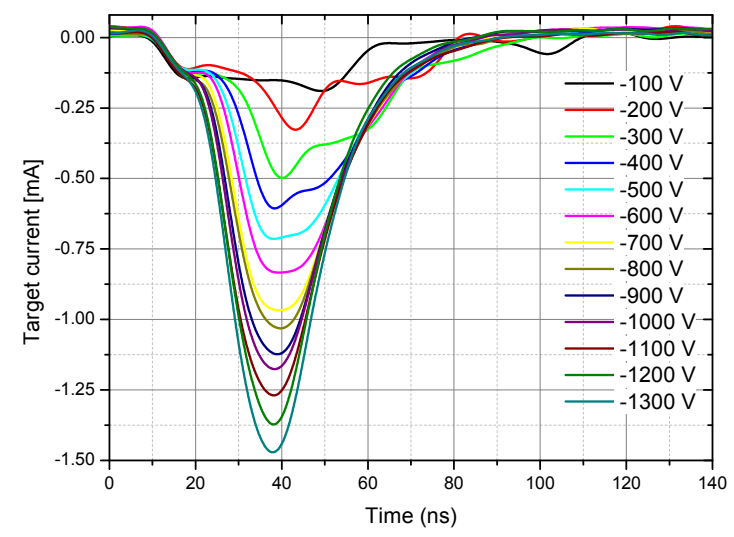

Figure 5. Dependence of ignition phase's negative TC on FC polarization. Bias voltages are reported in absolute value. Values obtained with a $0.5 \mathrm{~mm}$ target thickness. 
As a further clarification, we connected the input mesh to a negative bias larger than that on the FC, in order to understand if P2 increase due to electrons hitting the targets from the photopeak. Indeed, secondary electrons extracted from FC are suppressed using a small negative potential between the mesh and the FC plate, such that electrons extracted from energetic photons are pushed back to the metal. Such measurements confirmed the increase in P2's amplitude increasing (in absolute value) the bias on the input mesh (data not shown).

This eventually means that $\mathrm{P} 2$ is due to free electrons attracted by target's positive charging during the formation of P1. Moreover, this process is further enhanced by the electric field created in the space between target and FC. The cup (and/or the input mesh), indeed, is negatively polarized to attract positive ions, consequently a static electric field is superimposed to the transient fields developed during the laser ablation process. This static field is able to push negative charges towards the target, originating the observed current spectra. Indeed, considering the area enclosed by P1 and P2, it easy to obtain by numerical integration the total charge reaching the target in the first $14 \mathrm{~ns}$. Such values scale linearly with FC bias (Figure 6).

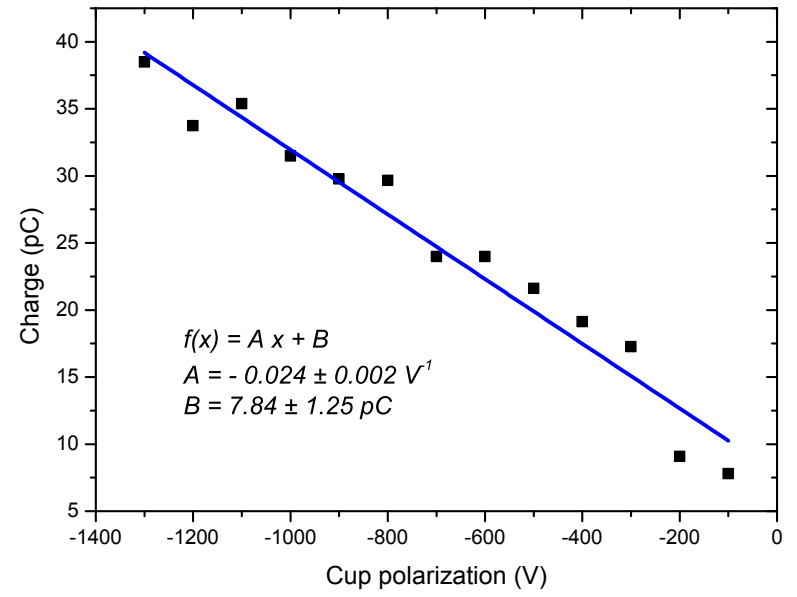

Figure 6. Charges reaching the target as a function of FC polarization. Ordinates are reported in absolute value.

The linear interpolation finds a $7.8 \mathrm{pC}$ peak charge in absence of any polarization. Such value is roughly the double one would expect if only P1 was present. Indeed, a rough extimation of the P1 area from Figure 3 for $0.5 \mathrm{~mm}$ thickness suggests it contributes for a charge near $3.0-4.0 \mathrm{pC}$. This confirms that charge unbalance on the target surface is able to attract electrons also in the absence of any electric field between $\mathrm{T}$ and FC. Consequently P2 exist irrespectively of any bias voltage on FC, as reported in [14]. This explains also because the "optimal thickness" affects also P2. In effect, if P1 increase in amplitude, a proportional increase should appear also for $\mathrm{P} 2$ in order to balance the excess positive charge on the target. It has, indeed, already pointed out that a target-to-FC static electric could influences the properties of ion emission [20]. Consequently, it is reasonable to suppose that the same applies also for TC.

\section{Conclusions}

We reported evidence on the negative target current observed in non-conductive materials during ignition phase. High resolution spectra of TC show that it is composed by a double peaked structure that strongly depends on target thickness. The first peak depends on the laser-matter interaction process, leading electrons from target to reach the conductive holder. This produces a net charge unbalance that further attracts free electrons on its surface. Furthermore, the amplitude of this second peak is readily enhanced by the presence of a strong electric field between target and Faraday cup.

\section{References}

[1] B. Chichkov, C. Momma, S. Nolte, F. von Alvensleben, A. Tünnermann, Appl Phys Mater Sci Process 63, 109 (1996)

[2] J.S. Pearlman, G.H. Dahlbacka, Applied Physics Letters 31, 414 (1977)

[3] R.F. Benjamin, G.H. McCall, A.W. Ehler, Phys. Rev. Lett. 42, 890 (1979)

[4] J.L. Borowitz, S. Eliezer, Y. Gazit, M. Givon, S. Jackel, A. Ludmirsky, D. Salzmann, E. Yarkoni, A. Zigler, B. Arad, Journal of Physics D: Applied Physics 20, 210 (1987)

[5] Y.A. Bykovskii, I.Y. Konyukhov, V.D. Peklenkov, Quantum Electronics 31, 45 (2001)

[6] A. Macchi, M. Borghesi, M. Passoni, Rev. Mod. Phys. 85, 751 (2013)

[7] J.L. Dubois, F. Lubrano-Lavaderci, D. Raffestin, J. Ribolzi, J. Gazave, A.C.L. Fontaine, E. d'Humières, S. Hulin, P. Nicolaï, A. Poyé et al., Phys. Rev. E 89, 013102 (2014)

[8] A. Poyé, J.L. Dubois, F. Lubrano-Lavaderci, E. D'Humières, M. Bardon, S. Hulin, M. BaillyGrandvaux, J. Ribolzi, D. Raffestin, J.J. Santos et al., Phys. Rev. E 92, 043107 (2015)

[9] J. Cikhardt, J. Krása, M. De Marco, M. Pfeifer, A. Velyhan, E. Krouský, B. Cikhardtová, D. Klír, K. Rezác, J. Ullschmied et al., Review of Scientific Instruments 85, 103507 (2014)

[10] M. De Marco, J. Krása, J. Cikhardt, M. Pfeifer, E. Krouský, D. Margarone, H. Ahmed, M. Borghesi, S. Kar, L. Giuffrida et al., Journal of Instrumentation 11, C06004 (2016)

[11] J. Krása, M. De Marco, J. Cikhardt, M. Pfeifer, A. Velyhan, D. Klír, K. Rezác, J. Limpouch, E. Krouský, J. Dostál et al., Plasma Physics and Controlled Fusion 59, 065007 (2017)

[12] M. De Marco, J. Cikhardt, J. Krása, A. Velyhan, M. Pfeifer, E. Krouskỳ, D. Klír, K. Řezáč, J. Limpouch, D. Margarone et al., Nukleonika 60, 239 (2015)

[13] J. Krása, E. Giuffreda, D. Delle Side, V. Nassisi, D. Klír, J. Cikhardt, K. Rezác, Laser and Particle Beams 35, 170-176 (2017) 
[14] J. Krása, D. Delle Side, E. Giuffreda, V. Nassisi, Laser and Particle Beams 33, 601-605 (2015)

[15] E. Giuffreda, D. Delle Side, J. Krása, V. Nassisi, Journal of Instrumentation 11, C05004 (2016)

[16] E. Giuffreda, D. Delle Side, V. Nassisi, J. Krása, Nuclear Instruments and Methods in Physics Research Section B: Beam Interactions with Materials and Atoms 406, 225 (2017)

[17] L. Velardi, M.V. Siciliano, D. Delle Side, V. Nassisi, Review of Scientific Instruments 83, $02 B 717$ (2012)
[18] V. Nassisi, L. Velardi, D. Delle Side, Applied Surface Science 272, 114 (2013)

[19] L. Velardi, D. Delle Side, J. Krása, V. Nassisi, Nuclear Instruments and Methods in Physics Research Section A: Accelerators, Spectrometers, Detectors and Associated Equipment 735, 564 (2014)

[20] M.M. Bialkowski, G.S. Hurst, J.E. Parks, D.H. Lowndes, G.E.J. Jr., Journal of Applied Physics 68, 4795 (1990) 